\section{Doenças crônicas e qualidade de vida na atenção primária à saúde}

\author{
Chronic diseases and quality of life in primary \\ health care
}

\section{${ }_{1}$ Universidade Católica de Pelotas, Pelotas, Brasil. \\ Correspondência \\ A. L.S. Azevedo \\ Universidade Católica de Pelotas. \\ Rua Paulo Marques 25, Pelotas, RS 96020-230, Brasil. anaazevedo03@hotmail.com}

\begin{abstract}
The aim of this cross-sectional study was to evaluate quality of life in chronic patients treated at primary care services associated with the Catholic University of Pelotas, Rio Grande do Sul State, Brazil. Quality of life was assessed by WHOQOLBref, and data on chronic diseases were obtained from patients' medical records and categorized as cardiovascular diseases, respiratory diseases, endocrine diseases, mental illness, and other musculoskeletal diseases. A total of 920 subjects were interviewed. Low self-rated quality of life was associated with female gender, older age, low socioeconomic status, single conjugal status, and chronic disease. In all domains of quality of life, mean levels were lower in patients with diseases of the nervous system, except in the environmental domains, which were lower in patients with mental illness. The results corroborate that quality of life in patients with chronic diseases is a priority for public health policies.
\end{abstract}

Chronic Disease; Quality of Life; Primary Health Care
Ana Lucia Soares de Azevedo ${ }^{1}$

Ricardo Azevedo da Silva 1

Elaine Tomasi 1

Luciana de Ávila Quevedo 1

\section{Resumo}

O objetivo do estudo é avaliar a qualidade de vida entre os usuários portadores de doenças crônicas que buscam atendimento nas unidades básicas de saúde (UBS) da Universidade Católica de Pelotas. Este trabalho consiste num estudo transversal aninhado a um estudo de intervenção na região sul do Rio Grande do Sul, Brasil. Para a avaliação da qualidade de vida foi utilizado o WHOQOL-BREF. Foram entrevistados 920 sujeitos. A percepção da qualidade de vida foi inferior em mulheres; pessoas mais velhas, de classe social baixa, que não viviam com companheiro, e com alguma doença crônica. Em todos os domínios da qualidade de vida, as médias mais baixas foram em pacientes com doenças do sistema nervoso, exceto no domínio Ambiente, que foi em pacientes com doenças mentais. A qualidade de vida dos indivíduos é um tema de fundamental importância e precisa ser colocado entre as prioridades das políticas públicas.

Doença Crônica; Qualidade de Vida; Atenção Primária à Saúde 


\section{Introdução}

O Sistema Único de Saúde (SUS) se encaminha para a sua consolidação por meio do Pacto pela Saúde, que aponta como prioridade, entre outras, a qualificação da atenção primária, definindo como modelo de atenção à saúde no Brasil a Estratégia Saúde da Família ${ }^{1}$. A atenção primária à saúde é definida como estratégia de organização do sistema de saúde para realizar ações de promoção à saúde, prevenção de agravos, diagnóstico, tratamento de problemas de saúde mais prevalentes e reabilitação individual e coletiva 2 . A atenção primária à saúde é a porta de entrada dos sistemas de saúde que oferece desafios especiais, pois muitos dos problemas trazidos pelos pacientes são geralmente vagos e não relacionados a doenças orgânicas específicas 3 .

Nesse contexto, estão inseridas as doenças crônicas que têm sido muito estudadas por conta da importância da busca de estratégias para redução da prevalência dos fatores de risco envolvidos, que estão diretamente relacionados às mudanças de estilo de vida e à qualidade de vida 4 . Historicamente, uma das definições de doença crônica mais amplamente aceita é aquela proposta em 1957 pela Comissão de Doenças Crônicas de Cambridge (Estados Unidos), na qual se incluíam todos os desvios do normal que tinham uma ou mais das seguintes características: permanência, presença de incapacidade residual, mudança patológica não reversível no sistema corporal, necessidade de treinamento especial do paciente para a reabilitação e previsão de um longo período de supervisão, observação e cuidados 5 .

A avaliação e o acompanhamento dos índices de qualidade de vida em pacientes com doenças crônicas têm sua utilidade no planejamento de estratégias de intervenção, já que fornecem informações importantes sobre o usuário, permitindo identificar suas prioridades e subsidiar os programas de saúde para que implementem ações efetivas e, assim, proporcionem melhor qualidade de vida aos usuários na atenção primária à saúde 6,7.

Avaliação da qualidade de vida é complicada pelo fato de não haver definição universalmente aceita. Qualidade de vida pode ser definida como a percepção do indivíduo de sua posição na vida, no contexto de sua cultura, no sistema de valores em que vive e em relação a suas expectativas, seus padrões e suas preocupações. O conceito incorpora a saúde física, o estado psicológico, o nível de independência, as relações sociais, as crenças pessoais e a relação com aspectos significativos do meio ambiente 8 . Em geral, pode ser influenciada direta e indireta- mente por fatores diversos relacionados à doença. Doenças crônicas tais como transtornos de ansiedade, depressão, insuficiência cardíaca, doenças isquêmicas do coração, hipertensão arterial, diabetes, enxaqueca, doença pulmonar obstrutiva crônica, asma e doenças osteomusculares estão associadas com a qualidade de vida diminuída 9,10,11,12.

A qualidade de vida em saúde é altamente discutida hoje. Alguns fatores que influenciam a avaliação da qualidade de vida já são conhecidos, como: sexo; idade, hábitos prejudiciais à saúde, como fumo e consumo de álcool; estado civil e pobreza $10,11,12$.

Assim, ainda existe necessidade de continuar pesquisando esse tema, sobretudo associado a doenças crônicas a fim de que se obtenham melhores resultados na prática clínica diária. Informações sobre a qualidade de vida de um indivíduo podem ser usadas para monitorar o seu processo de tratamento, comparar diferentes estágios da doença e facilitar a tomada de decisão clínica e cuidados em saúde 13. Portanto, este estudo teve como objetivo avaliar a qualidade de vida entre os usuários portadores de doenças crônicas (cardiovasculares, respiratórias, endócrinas, mentais e osteomusculares) que buscaram atendimento nas unidades básicas de saúde (UBS) da Universidade Católica de Pelotas (UCPel).

\section{Métodos}

Trata-se de um estudo transversal aninhado a um estudo de intervenção em três UBS vinculadas à UCPel (UBS Fátima, UBS CAIC Pestano e UBS Santa Terezinha) na zona sul do Rio Grande do Sul.

A amostra foi de conveniência e incluiu pacientes das três UBS que buscaram algum tipo de atendimento nos dias pares entre 1o de março e 30 de julho de 2009. Foram considerados elegíveis indivíduos maiores de 14 anos e que fossem moradores da área de abrangência da UBS. No período estipulado, foram registrados 1.516 atendimentos nas três UBS, porém muitos pacientes que consultaram não moravam na área de abrangência, muitas vezes dando endereços inexistentes para conseguir consulta; desse modo, foi possível incluir 1.081 sujeitos.

Os dados de identificação foram retirados do prontuário do paciente por ocasião do atendimento na UBS. Para a coleta de dados foi utilizado um questionário com variáveis sobre características sociodemográficas (como, sexo, idade, escolaridade e classe econômica) e comportamentais. Os questionários foram aplicados por alunos dos cursos da área da saúde, capacitados 
para realizarem as entrevistas, nas residências dos pacientes.

A presença de doenças crônicas foi retirada do código CID registrado pelo médico no prontuário do paciente, sendo categorizadas em doenças cardiovasculares, doenças respiratórias, doenças endócrinas, doenças mentais, doenças osteomusculares e outras (doenças digestivas, sistema geniturinário, aparelho da visão, infecciosas e neoplasias), as quais foram agrupadas por serem de baixa prevalência nessa amostra. Em razão do fato de alguns pacientes terem o registro de mais de uma doença crônica, e a dificuldade metodológica em estabelecer qual a principal doença, foi utilizado como critério o CID da doença crônica atual - motivo da consulta.

Para a avaliação da qualidade de vida, foi utilizado o WHOQOL-BREF, que é uma versão abreviada do WHOQOL-100, instrumento criado pelo Grupo WHOQOL específico para avaliar qualidade de vida. Tal avaliação se concentra no que é percebido pelos entrevistados, e não um meio de medição de todos os sintomas de forma detalhada, doenças ou condições, mas os efeitos das intervenções da doença e saúde na qualidade de vida. O WHOQOL-BREF é composto por 26 questões, com 24 facetas relacionadas à qualidade de vida e agrupadas em quatro domínios: saúde física, psicológica, relações sociais e meio ambiente. No domínio físico, estão incorporadas as facetas, atividades da vida diária, dependência de substâncias medicinais e ajuda médica, energia e fadiga, mobilidade, dor e desconforto, sono e repouso e capacidade de trabalho. No domínio psicológico, estão incluídas imagem corporal e aparência, sentimentos negativos e positivos, autoestima, espiritualidade/religião/crenças pessoais e pensamento, aprendizagem, memória e concentração. No domínio relações sociais, estão incluídas nas facetas as relações pessoais, apoio social e atividade sexual. E no domínio ambiente estão incluídas as facetas recursos financeiros, segurança, liberdade e segurança física, cuidados de saúde e sociais: disponibilidade e qualidade do ambiente doméstico, oportunidades para adquirir novas informações e habilidades, participação e oportunidades de recreação/lazer, ambiente físico (poluição/ruído/trânsito/ clima) e transporte. A avaliação de qualidade de vida é verificada pelas médias que variam de 26 a 130 pontos e de 0 a 100 para cada domínio, não existindo um ponto de corte que indique médias desejáveis, contudo, quanto mais alta as médias, melhor percepção da qualidade de vida tem o indivíduo 14,15,16,17.

A classificação econômica foi avaliada com os critérios da Associação Brasileira de Empresas de Pesquisa (ABEP. Critério de classificação eco- nômico no Brasil. http://www.abep.org/codigos guias/ABEP_CCEB.pdf, acessado em Set/2011). Essa classificação é baseada no acúmulo de bens materiais e na escolaridade do chefe da família, classificando os sujeitos em cinco níveis (A, B, C, $\mathrm{D}$ e E). A idade dos indivíduos, por incluir uma ampla faixa etária, foi categorizada em tercil, ficando dividida em 14-38 anos, 39-56 anos e 57 anos ou mais.

Para análise dos dados, foi realizada a frequência das características da amostra, comparação entre médias (teste t e ANOVA) e regressão linear simples e ajustada. Foi proposto um modelo conceitual hierarquizado para análise dos dados, sendo o primeiro nível composto por variáveis sociodemográficas (sexo, idade, classificação econômica e viver com companheiro) e o segundo por variáveis comportamentais e variáveis relacionadas à saúde (uso de tabaco, uso de álcool e doença crônica). As variáveis que apresentaram $\mathrm{p}<0,20$ foram para a análise ajustada conforme os níveis propostos pelo modelo hierárquico ${ }^{18}$. Foi realizada análise de resíduos, e na regressão linear de cada domínio pode-se ver que os resíduos tiveram distribuição normal e a variância dos mesmos foi constante. A análise dos dados foi realizada no programa SPSS 13.0 (SPSS Inc., Chicago, Estados Unidos).

Este estudo foi aprovado pelo Comitê de Ética em Pesquisa da UCPel, de acordo com a Resolução no 196/96 do Conselho Nacional de Saúde, conforme protocolo no 2009/19.

\section{Resultados}

Foram entrevistados 1.081 sujeitos. Desses, 14,9\% não tinham nenhum diagnóstico registrado no prontuário, nem atual nem passado, totalizando uma amostra de 920 entrevistados.

Quanto às características da amostra, 668 $(72,6 \%)$ eram mulheres, com idade variando entre 14 a 96 anos, sendo a média de 46,3 anos (DP = $18,6)$. A maioria dos sujeitos $(60,6 \%)$ era da classe econômica C e 488 (53,3\%) viviam com companheiro. Quanto às doenças crônicas, a prevalência foi de $51,8 \%$, sendo 276 (30\%) com doenças circulatórias, seguidas de doenças mentais (7\%), doenças endócrinas $(4,7 \%)$, doenças osteomusculares $(4,2 \%)$, doenças respiratórias $(2,1 \%)$, doenças do sistema nervoso (1,3\%) e outras doenças crônicas $(2,6)$ (Tabela 1). Em relação à qualidade de vida, as médias foram as seguintes: aspecto físico $(65,1 ; \mathrm{DP}=20,9)$, aspecto psicológico $(67,1$; $\mathrm{DP}=17,3)$, relações sociais $(71,3 ; \mathrm{DP}=17,7)$ e ambiente $(59,0 ; \mathrm{DP}=14,2)$ (Tabela 2).

$\mathrm{Na}$ análise bruta, as médias do domínio aspecto físico foram menores em mulheres $(p=$ 
Tabela 1

Distribuição da amostra de acordo com as características de pacientes atendidos em três unidades básicas de saúde (UBS) em Pelotas, Rio Grande do Sul, Brasil.

\begin{tabular}{|c|c|c|}
\hline Variáveis & $\mathbf{n}$ & $\%$ \\
\hline \multicolumn{3}{|l|}{ Sexo } \\
\hline Masculino & 252 & 27,4 \\
\hline Feminino & 668 & 72,6 \\
\hline \multicolumn{3}{|l|}{ Idade (anos) } \\
\hline $14-36$ & 301 & 33,0 \\
\hline $37-56$ & 300 & 32,9 \\
\hline 57 ou mais & 310 & 34,0 \\
\hline \multicolumn{3}{|c|}{ Classificação socioeconômica } \\
\hline$A \in B$ & 92 & 10,1 \\
\hline C & 552 & 60,6 \\
\hline$D$ e $E$ & 267 & 29,3 \\
\hline \multicolumn{3}{|c|}{ Viver com companheiro } \\
\hline Não & 425 & 46,5 \\
\hline Sim & 488 & 53,3 \\
\hline \multicolumn{3}{|l|}{ Doença crônica } \\
\hline Nenhuma & 443 & 48,2 \\
\hline Circulatória & 276 & 30,0 \\
\hline Respiratória & 19 & 2,1 \\
\hline Nervoso & 12 & 1,3 \\
\hline Osteomuscular & 39 & 4,2 \\
\hline Endócrino & 43 & 4,7 \\
\hline Mental & 64 & 7,0 \\
\hline Outros & 24 & 2,6 \\
\hline Total & 920 & 100,0 \\
\hline
\end{tabular}

Tabela 2

Distribuição da amostra de acordo com as médias de qualidade de vida de pacientes atendidos em três unidades básicas de saúde (UBS) em Pelotas, Rio Grande do Sul, Brasil.

\begin{tabular}{lll}
\hline Variáveis & Média & DP \\
\hline Aspecto físico & 65,05 & 20,88 \\
Aspecto psicológico & 67,10 & 17,30 \\
Relações sociais & 71,33 & 17,71 \\
Ambiente & 59,01 & 14,23
\end{tabular}

DP: desvio-padrão.

0,010), indivíduos que não viviam com companheiro ( $\mathrm{p}=0,009)$, e tinham alguma doença crônica $(\mathrm{p}<0,001)$. As variáveis idade e classe econômica também se mostraram associadas com o domínio ( $\mathrm{p}<0,001$ e $\mathrm{p}<0,001)$, e aparentemente as médias da categoria 57 anos ou mais e classes D e E foram as menores. No domínio psicológico, as médias mais baixas foram entre as mulheres ( $p=0,016)$, pacientes que não viviam com companheiro $(\mathrm{p}=0,003)$ e tinham alguma doença crônica ( $p=0,002)$. As variáveis idade e classe econômica também se mostraram associadas ( $\mathrm{p}$ $=0,001$ e $\mathrm{p}<0,001)$, e aparentemente as médias da categoria entre 37 e 56 anos e classes D e E foram as menores. Quanto ao domínio relações sociais, as médias mais baixas foram em pacientes que não viviam com companheiro $(\mathrm{p}<0,001)$ e que tinham alguma doença crônica $(\mathrm{p}=0,010)$. As variáveis idade e classe econômica também se mostraram associadas ( $\mathrm{p}=0,018$ e $\mathrm{p}<0,001)$, e aparentemente as médias da categoria entre 37 e 56 anos e classes D e E foram as menores. As médias de qualidade de vida no domínio ambiente foram inferiores em quem não vivia com companheiro $(\mathrm{p}<0,001)$ e quem tinha alguma doença crônica ( $p=0,007)$. A variável classe econômica também foi associada a esse domínio, e aparentemente indivíduos das classes D e E ( $\mathrm{p}<$ 0,001 ) tiveram as médias mais baixas (Tabela 3 ).

A Tabela 4 mostra as médias nos quatro domínios da qualidade de vida para cada grupo das doenças crônicas. Pessoas com doenças do sistema nervoso tiveram médias inferiores nos domínios aspecto físico, psicológico e relações sociais. No domínio ambiente, as médias foram menores em indivíduos com doenças mentais (Tabela 4).

A presença de doença crônica no domínio físico foi ajustada para sexo, idade, classe econômica, viver com companheiro; no domínio psicológico para sexo, idade, classe econômica, viver com companheiro; no domínio relações sociais, para sexo, idade, classe econômica, viver com companheiro; e no domínio ambiente, para idade, classe econômica e viver com companheiro.

Após a análise ajustada, permaneceram associadas ao domínio físico as variáveis sexo ( $\mathrm{p}=$ $0,010)$; idade $(\mathrm{p}<0,001)$; classificação econômica ( $\mathrm{p}<0,001)$ e doenças crônicas $(\mathrm{p}=0,005)$. Assim, homens tiveram 3,9 (IC95\%: 1,0; 7,0) pontos a mais na média no domínio físico do que as mulheres. Quanto à idade, a cada categoria a média diminuiu 7,8 (IC95\%: -9,4; -6,3) pontos, sendo as médias mais altas em pessoas mais jovens. Quanto à classe econômica, as médias diminuíram 6,5 (IC95\%: -9,3; -3,6) pontos a cada categoria da ABEP, sendo as maiores médias nas classes mais altas, e indivíduos com doença crônica tiveram 3,9 (IC95\%: -6,6; -1,2) pontos a menos na média do domínio físico do que os que não tinham doença crônica. Em relação ao domínio psicológico, as variáveis que permaneceram associadas foram sexo $(p=0,016)$; classifi- 
Tabela 3

Médias dos domínios do WHOQOL-BREF de acordo com as variáveis de exposição em pacientes atendidos em três unidades básicas de saúde (UBS) em Pelotas, Rio Grande do Sul, Brasil.

\begin{tabular}{|c|c|c|c|c|c|c|c|c|}
\hline \multirow[t]{2}{*}{ Variáveis } & \multicolumn{2}{|c|}{ Aspecto físico } & \multicolumn{2}{|c|}{ Aspecto psicológico } & \multicolumn{2}{|c|}{ Relações sociais } & \multicolumn{2}{|c|}{ Ambiente } \\
\hline & Média (DP) & Valor de $p$ & Média (DP) & Valor de p & Média (DP) & Valor de p & Média (DP) & Valor de $\mathrm{p}$ \\
\hline Sexo & & 0,010 * & & 0,016 * & & 0,068 & & 0,225 \\
\hline Masculino & $67,9(21,5)$ & & $69,3(17,1)$ & & $73,1(17,1)$ & & $60,0(15,2)$ & \\
\hline Feminino & $63,9(20,6)$ & & $66,3(17,3)$ & & $70,7(17,9)$ & & $58,7(13,8)$ & \\
\hline $14-36$ & $74,5(17,3)$ & & $70,2(16,6)$ & & $73,8(19,5)$ & & $61,0(14,7)$ & \\
\hline $37-56$ & $62,7(21,6)$ & & $65,3(18,4)$ & & $69,5(17,9)$ & & $56,7(14,3)$ & \\
\hline 57 ou mais & $57,9(19,9)$ & & $65,7(16,5)$ & & $70,6(15,3)$ & & $59,2(13,5)$ & \\
\hline Classificação econômica & & $<0,001 *$ & & $<0,001 *$ & & $<0,001$ * & & $<0,001$ * \\
\hline$A$ e $B$ & $74,2(18,9)$ & & $75,0(13,5)$ & & $79,3(15,9)$ & & $67,6(12,7)$ & \\
\hline C & $66,8(20,0)$ & & $68,2(16,8)$ & & $71,8(17,2)$ & & $59,7(13,9)$ & \\
\hline Viver com companheiro & & 0,009 * & & 0,003 * & & $<0,001 *$ & & $<0,001$ * \\
\hline Não & $63,1(21,9)$ & & $65,3(18,5)$ & & $68,3(18,9)$ & & $57,0(14,7)$ & \\
\hline Sim & $66,7(19,8)$ & & $68,7(16,0)$ & & $73,9(16,2)$ & & $60,7(13,5)$ & \\
\hline Doença crônica & & $<0,001 *$ & & 0,002 * & & 0,010 * & & $0,007 *$ \\
\hline Não & $69,6(20,1)$ & & $68,9(17,2)$ & & $72,9(17,8)$ & & $60,3(14,3)$ & \\
\hline Sim & $60,8(20,7)$ & & $65,4(17,2)$ & & $69,9(17,5)$ & & $57,8(14,1)$ & \\
\hline Total & $65,1(20,8)$ & & $67,1(17,3)$ & & $71,3(17,7)$ & & $59,0(14,2)$ & \\
\hline
\end{tabular}

DP: desvio-padrão.

Nota: para comparação de dois grupos, foi utilizado o teste t e a Anova para comparação de três ou mais grupos.

* Diferença estatisticamente significativa entre as médias.

Tabela 4

Médias dos domínios do WHOQOL-BREF de acordo com as doenças crônicas de pacientes atendidos em três unidades básicas de saúde (UBS) em Pelotas, Rio Grande do Sul, Brasil.

\begin{tabular}{|c|c|c|c|c|c|c|c|c|}
\hline \multirow[t]{2}{*}{ Variáveis } & \multicolumn{2}{|c|}{ Aspecto físico } & \multicolumn{2}{|c|}{ Aspecto psicológico } & \multicolumn{2}{|c|}{ Relações sociais } & \multicolumn{2}{|c|}{ Ambiente } \\
\hline & Média (DP) & Valor de $p$ & Média (DP) & Valor de $p$ & Média (DP) & Valor de $p$ & Média (DP) & Valor de $p$ \\
\hline Nenhuma & $69,6(20,1)$ & & $68,9(17,2)$ & & $72,9(17,8)$ & & $60,3(17,8)$ & \\
\hline Circulatória & $60,5(20,4)$ & & $66,9(16,7)$ & & $71,2(15,8)$ & & $58,8(13,3)$ & \\
\hline Respiratória & $65,1(20,0)$ & & $66,4(13,6)$ & & $63,6(17,4)$ & & $55,2(8,4)$ & \\
\hline Nervoso & $57,1(25,0)$ & & $58,7(20,1)$ & & $60,1(18,4)$ & & $53,4(16,1)$ & \\
\hline Osteomuscular & $61,2(21,6)$ & & $67,0(16,1)$ & & $74,6(18,3)$ & & $60,7(14,5)$ & \\
\hline Endócrino & $62,2(19,2)$ & & $66,9(17,2)$ & & $69,0(21,4)$ & & $60,3(17,4)$ & \\
\hline Mental & $60,7(21,7)$ & & $59,4(19,3)$ & & $65,6(18,2)$ & & $52,2(12,8)$ & \\
\hline Outros & $61,2(22,5)$ & & $61,6(18,2)$ & & $69,4(20,8)$ & & $55,9(16,4)$ & \\
\hline
\end{tabular}

DP: desvio-padrão.

* Diferença estatisticamente significativa entre as médias. 
cação econômica $(\mathrm{p}<0,001)$ e doenças crônicas ( $p=0,062$ ). Homens tiveram 3,1 (IC95\%: 0,6; 5,6) pontos a mais na média do domínio psicológico comparado às mulheres. Quanto à classe econômica, as médias diminuíram 5,3 (IC95\%: -7,8; $-2,8$ ) pontos a cada categoria da ABEP, sendo as médias mais altas nas classes mais altas. As médias aumentaram 2,3 (IC95\%: -4,7; 0,1) pontos em indivíduos com doenças crônicas. No domínio de relações sociais, as variáveis associadas às médias de qualidade de vida foram classificação econômica ( $p=0,005)$ e viver com companheiro $(\mathrm{p}<0,001)$. A cada classe social mais baixa, as médias diminuíram 3,6 (IC95\%: -6,3; -1,1) pontos. Aqueles que viviam com companheiro tiveram 4,6 (IC95\%: 2,$3 ; 7,0$ ) pontos a mais na média no domínio relações sociais do que aqueles que não viviam com um companheiro.

Quanto ao domínio ambiente, as variáveis que permaneceram associadas foram classificação econômica ( $\mathrm{p}<0,001)$; viver com companheiro $(p=0,003)$ e presença de doenças crônicas $(\mathrm{p}=0,006)$. Quanto à classe econômica, as médias diminuíram 5,3 (IC95\%: -7,4; -3,3) pontos a cada categoria da ABEP, sendo as médias maiores nas classes mais altas. Pacientes que viviam com companheiro tiveram 2,8 (IC95\%: 0,9; 4,6) pontos a mais na média do domínio ambiente do que aqueles que não viviam com companheiro. Quanto às doenças crônicas, as médias diminuíram 2,1 (IC95\%: -4,1; -1,1) pontos, em pacientes com alguma doença crônica (Tabela 5).

\section{Discussão}

O presente estudo encontrou que, na amostra analisada, a percepção da qualidade de vida foi inferior em mulheres; pessoas mais velhas, de classe social baixa, que não viviam com companheiro e com alguma doença crônica. Em todos os domínios da qualidade de vida, as médias mais baixas foram em pacientes com doenças do sistema nervoso, exceto no domínio ambiente, que foi em pacientes com doenças mentais.

As pessoas que sofrem de diferentes doenças crônicas têm de aprender a conviver com várias limitações em seu cotidiano, uma vez que a doença exige adaptação do paciente e dos familiares em diferentes aspectos da vida, e geralmente tais mudanças se desdobram com o tempo 19. Esse ajustamento implica, possivelmente, uma marcada diminuição da qualidade de vida.

A relação entre doenças do sistema nervoso e médias mais baixas de qualidade de vida pode ser devido aos efeitos diretos da própria doença e os efeitos colaterais do tratamento 20 . Sabe-se que os principais sinais e sintomas das doenças do sistema nervoso são motores e que eles, somados ao sedentarismo e ao isolamento social que essas doenças costumam provocar, interferem significativamente na percepção da qualidade de vida dos pacientes 21 .

Somente no domínio ambiente, que as doenças mentais tiveram médias inferiores às doenças do sistema nervoso. O domínio ambiente envolve insegurança, ambiente físico menos saudável, menos disponibilidade de dinheiro, menos acesso à informação e lazer e menor satisfação com a moradia, serviços de saúde e meios de transporte 17. Logo, tal associação pode ser justificada pelo fato de que transtornos mentais comuns podem ser corriqueiramente encontrados em indivíduos com baixa classe econômica, baixa escolaridade, menor número de bens, condições precárias de moradia e desemprego 22,23.

Estudos sobre qualidade de vida em pacientes com doenças crônicas demonstram que as mulheres em geral as percebem de forma pior que os homens 24,25; essa relação se evidenciou no presente estudo. É importante considerar que as mulheres buscam mais atendimentos nos serviços de saúde, e uma possível justificativa é o fato de que além de terem uma autopercepção pior de saúde do que os homens expressam com maior facilidade seus sintomas, realizam pré-natal e consultas de puericultura. Sabe-se também que, embora as mulheres tenham melhores taxas de sobrevivência do que homens em todo o ciclo de vida, elas experimentam taxas mais elevadas de limitações de morbidade e funcional 26.

Em relação à idade, pacientes mais velhos apresentaram médias inferiores de qualidade de vida no aspecto físico. Esse aspecto é central da qualidade de vida do idoso, que sofre influências com o aumento da idade, conforme já demonstrado em outros estudos 27,28,29.

Verificou-se também que pacientes de classes sociais mais baixas demonstraram pior qualidade de vida nos quatro domínios. É relevante destacar que, no Brasil, a desigualdade na distribuição de renda, o analfabetismo, o baixo grau de escolaridade, as condições de habitação e o ambiente precário causam impactos negativos sobre a qualidade de vida e a saúde dos sujeitos 14,30 .

Os sujeitos que não viviam com companhei-ro(a) também tiveram percepções de qualidade de vida inferior. Pode-se depreender que a falta de um parceiro pode estar relacionada a sentimentos de solidão e de isolamento e, portanto, a menor bem-estar. Pessoas que vivem com companheiro dispõem de maior suporte (familiar/ social) 22.

Deve-se ressaltar como limitação deste estudo que os diagnósticos foram retirados dos regis- 
Tabela 5

Análise ajustada das médias dos domínios do WHOQOL-BREF de acordo com as variáveis de exposição em pacientes atendidos em três unidades básicas de saúde (UBS) em Pelotas, Rio Grande do Sul, Brasil.

\begin{tabular}{|c|c|c|c|c|c|c|c|c|}
\hline \multirow[t]{2}{*}{ Variáveis } & \multicolumn{2}{|c|}{ Aspecto físico } & \multicolumn{2}{|c|}{ Aspecto psicológico } & \multicolumn{2}{|c|}{ Relações sociais } & \multicolumn{2}{|c|}{ Ambiente } \\
\hline & Média (IC95\%) & Valor de $p$ & Média (IC95\%) & Valor de $p$ & Média (IC95\%) & Valor de $p$ & Média (IC95\%) & Valor de $p$ \\
\hline Sexo (masculino) & $3,9(1,0 ; 7,0)$ & $0,010 *$ & $3,1(0,6 ; 5,6)$ & 0,016 * & $2,4(-0,2 ; 4,9)$ & 0,068 & & \\
\hline Idade (57 ou mais) & $-7,8(-9,4 ;-6,3)$ & $<0,001 *$ & $-1,2(-2,7 ; 0,3)$ & 0,097 & $-0,8(-2,3 ; 0,7)$ & 0,282 & $-0,1(-1,2 ; 1,2)$ & 0,947 \\
\hline Classificação econômica (D e E) & $-6,5(-9,3 ;-3,6)$ & $<0,001 *$ & $-5,3(-7,8 ;-2,8)$ & $<0,001 *$ & $-3,6(-6,3 ;-1,1)$ & 0,005 * & $-5,3(-7,4 ;-3,3)$ & $<0,001 *$ \\
\hline Viver com companheiro & $2,2(-0,4 ; 4,8)$ & 0,088 & $2,2(-0,7 ; 4,5)$ & 0,058 & $4,6(2,3 ; 7,0)$ & $<0,001 *$ & $2,8(0,9 ; 4,6)$ & 0,003 * \\
\hline Doença crônica & $-3,9(-6,6 ;-1,2)$ & 0,005 * & $-2,3(-4,7 ; 0,1)$ & 0,062 & $-2,3(-4,8 ; 0,1)$ & 0,063 & $-2,1(-4,1 ;-0,1)$ & 0,006 * \\
\hline
\end{tabular}

IC95\%: intervalo de $95 \%$ de confiança.

* Diferença estatisticamente significativa entre as médias.

tros dos prontuários (diagnóstico realizado pelo médico da atenção primária) sem reavaliar se os diagnósticos estavam corretos. Outra limitação se refere à ordem da cadeia causal, pois, por ser um estudo transversal, não é possível identificar se a qualidade de vida é diminuída em quem tem doenças crônicas, ou se as doenças crônicas aparecem em pessoas que já apresentavam qualidade de vida diminuída. Outra limitação a ser destacada se refere à dificuldade em estabelecer se o paciente tinha uma ou mais doenças crônicas. É provável que muitos deles possam ter mais de um diagnóstico, todavia, devido à falha no registro dos prontuários, foi impossível realizar tal análse. Mesmo assim, ficou evidente que ao menos a presença de uma doença crônica já afeta a qualidade de vida dos pacientes da amostra.

No entanto, este estudo demonstrou que existe uma relação entre qualidade de vida e presença de doença crônica. A medida da qualidade de vida gera informações que podem ser usadas para rastreamento e identificação das necessidades de saúde de uma população, decisão sobre as prioridades em assistir a determinados setores, alocação de recursos e comparação dos estados de saúde de diferentes tipos de tratamentos realizados 3,20,23,31.
As metas de atendimento de pacientes com uma condição crônica são melhorar o seu estado funcional, minimizar os sintomas, controlar a dor, reduzir a deficiência, e prolongar a vida mediante prevenção secundária 32 . Os médicos de família, para atingirem as metas nos cuidados de pacientes com comorbidades, precisam desenvolver uma parceria com os pacientes e estabelecer prioridades para atender às necessidades deles com o objetivo de manter uma boa qualidade de vida 8,9 .

O desenvolvimento e a necessidade de se investir em programas na atenção primária, que é a porta de entrada do sistema de saúde, devem receber mais destaque no SUS. A atenção primária é apenas um componente (embora seja o componente fundamental) dos sistemas de saúde, e tem o objetivo de oferecer acesso universal e serviços abrangentes, coordenar e expandir a cobertura para níveis mais complexos do cuidado 13. A qualidade de vida dos indivíduos parece ser um tema de fundamental importância e precisa ser colocado entre as prioridades das políticas públicas. 


\section{Resumen}

El objetivo de este estudio de la Universidad Católica de Pelotas es evaluar la calidad de vida de los usuarios con enfermedades crónicas que buscan atención en las unidades básicas de salud (UBS). Este trabajo es un estudio transversal, anidado en un estudio de intervención en la región sur de Rio Grande do Sul, Brasil. Para evaluar la calidad de vida se utilizó el WHOQOL-BREF. Entrevistamos a 920 individuos. La percepción de la calidad de vida fue menor en las mujeres, los adultos mayores, los de clase social baja, los que no vivían con su pareja y los que tenían alguna enfermedad crónica. En todos los dominios de la calidad de vida, los promedios fueron menores en los pacientes con enfermedades del sistema nervioso, excepto en el dominio ambiente en pacientes con enfermedad mental. La calidad de vida de las personas es un tema de importancia fundamental y debe estar situado entre las prioridades de las políticas públicas.

Enfermedad Crónica; Calidad de Vida; Atención Primaria de Salud

\section{Colaboradores}

A concepção e a organização do artigo foram feitas com a participação de todos os autores. A. L. S. Azevedo e L. A. Quevedo foram responsáveis pela formulação e elaboração do projeto, incluindo o desenho da pesquisa, revisão bibliográfica, análise dos dados, interpretação e apresentação dos resultados.

\section{Referências}

1. Ministério da Saúde. Diretrizes operacionais dos Pactos pela Vida, em Defesa do SUS e de Gestão. Brasília: Ministério da Saúde; 2006. (Série A. Normas e Manuais Técnicos).

2. Facchini LA, Piccini RX, Tomasi E, Thumé E, Teixeira VA, Silveira DS, et al. Avaliação de efetividade da Atenção Básica à Saúde em municípios das regiões Sul e Nordeste do Brasil: contribuições metodológicas. Cad Saúde Pública 2008; 24 Suppl 1:S159-72.

3. Lima AFBS, Fleck MPA. Quality of life, diagnosis, and treatment of patients with major depression: a prospective cohort study in primary care. Rev Bras Psiquiatr 2011; 33:245-51.

4. Capilheira M, Santos IS. Doenças crônicas não transmissíveis: desempenho no cuidado médico em atenção primária à saúde no sul do Brasil. Cad Saúde Pública 2011; 27:1143-53.

5. Kuller L, Tonascia S. A follow-up study of the Commission on Chronic Illness morbidity survey in Baltimore. IV. Factors influencing mortality from stroke and arteriosclerotic heart disease (19541967). J Chronic Dis 1971; 24:111-24.
6. Kluthcovsky AC, Kluthcovsky FA. OWHOQOL-Bref, um instrumento para avaliar qualidade de vida: uma revisão sistemática. Rev Psiquiatr Rio Gd Sul 2009; 31(3 Suppl):0-0.

7. Skevington SM, Lotfy M, O'Connel KA. The World Health Organization's WHOQOL-BREF quality of life assessment: psychometric properties and results of the international field trial a report from the WHOQOL Group. Qual Life Res 2004; 13:299310.

8. Fleck MPA. A avaliação de qualidade de vida: guia para profissionais da saúde. Porto Alegre: Editora Artmed; 2008.

9. Sprangers MA, de Regt EB, Andries F, van Agt HM, Bijl RV, de Boer JB, et al. Which chronic conditions are associated with better or poorer quality of life? J Clin Epidemiol 2000; 53:895-907.

10. Grumbach K. Chronic illness, comorbidities, and the need for medical generalism. Ann Fam Med 2003; 1:4-7. 
11. Lim LZ, Jin A, Ng TP. Anxiety and depression, chronic physical conditions, and quality of life in an urban population sample study. Soc Psychiatry Psychiatr Epidemiol 2012; 47:1047-53.

12. Fortin M. Relationship between multimorbidity and health-related quality of life of patients in primary care. Qual Life Res 2006; 15:83-91.

13. Skevington SM, McCrate FM. Expecting a good quality of life in health: assessing people with diverse diseases and conditions using the WHOQOLBREF. Health Expect 2012; 15:49-62.

14. Minayo MCS, Hartz ZMA, Buss PM. Qualidade de vida e saúde: um debate necessário. Ciênc Saúde Coletiva 2000; 5:7-18.

15. Corso AN, Costa LS, Fleck MPA, Heldt E. Impacto de sintomas depressivos na qualidade de vida de usuários da rede básica de saúde. Rev Gaúch Enferm 2000; 30:257-62.

16. Gonçalves DM, Kapczinski F. Prevalência de transtornos mentais em indivíduos de uma unidade de referência para Programa Saúde da Família em Santa Cruz do Sul, Rio Grande do Sul, Brasil. Cad Saúde Pública 2008; 24:2043-53.

17. World Health Organization. WHOQOL-BREF: instructions. http://www.who.int/mental_health/ media/en/76.pdf (acessado em Set/2011).

18. Victora CG, Huttly SR, Fuchs SC, Olinto MT. The role of conceptual frameworks in epidemiological analysis: a hierarchical approach. Int J Epidemiol 1997; 26:224-7.

19. Stanton AL, Revenson TA, Tennen H. Health psychology: psychological adjustment to chronic disease. Ann Rev psychol 2007; 58:565-92.

20. Baune BT, Aljeesh Y. The association of psychological stress and health related quality of life among patients with stroke and hypertension in Gaza Strip. Ann Gen Psychiatry 2006; 5:6.

21. Lana RC, Álvares LMRS, Nasciutti-Prudente C, Goulart FRP, Teixeira-Salmela LF, Cardoso FE. Percepção da qualidade de vida de indivíduos com doença de Parkinson através do PDQ-39. Rev Bras Fisioter 2007; 11:397-402.

22. Costa AG, Ludermir AB. Transtornos mentais comuns e apoio social: estudo em comunidade rural da Zona da Mata de Pernambuco, Brasil. Cad Saúde Pública 2005; 21:73-9.
23. Lima MS, Béria JU, Tomasi E, Conceição AT, Mari JJ. Stressful life events and minor psychiatric disorders: an estimate of the population attributable fraction in a Brazilian community-based study. Int J Psychiatry Med 1996; 26:211-22.

24. Mrus JM, Williams PL, Tsevat J, Cohn SE, Wu AW. Gender differences in health related quality of life in patients with HIV/AIDS. Qual Life Res 2005; 14:479-91.

25. Teh CF, Kilbourne AM, McCarthy JF, Welsh D, Blow FC. Gender differences in health related quality of life for veterans with serious mental illness. Psychiatr Serv 2008; 59:663-9.

26. Verbrugge LM. The twain meet: empirical explanations of sex differences in health and mortality. J Health Soc Behav 1989; 30:282-304.

27. Alves LC, Leimann BCQ, Vasconcelos MEL, Carvalho MS, Vasconcelos AGG, Fonseca TCO, et al. A influência das doenças crônicas na capacidade funcional dos idosos do Município de São Paulo, Brasil. Cad Saúde Pública 2007; 23:1924-30.

28. Paschoal SMP. Qualidade de vida na velhice. In: Freitas EV, Xavier FA, organizadores. Tratado de geriatria e gerontologia. 3a Ed. Rio de Janeiro: Editora Guanabara Koogan; 2011. p. 99-106.

29. Barbosa AR, Souza JMP, Lebrão ML, Laurenti R, Marucci MFN. Functional limitations of Brazilian elderly by age and gender differences: data from SABE Survey. Cad Saúde Pública 2005; 21:1177-85.

30. Buss PM. Promoção da saúde e qualidade de vida. Ciênc Saúde Coletiva 2000; 5:163-77.

31. Fortin M, Dubois M-F, Hudon C, Soubhi H, Almirall J. Multimorbidity and quality of life: a closer look. Health Qual Life Outcomes 2007; 5:52.

32. Bossola M, Murri R, Onder G, Turriziani A, Fantoni M, Padua L. Physicians' knowledge of health-related quality of life and perception of its importance in daily clinical practice. Health Qual Life Outcomes 2010; 8:43.

Recebido em 11/Set/2012

Versão final reapresentada em 23/Mar/2013

Aprovado em 03/Abr/2013 